\title{
Strategies for Biochemical and Pathologic Quality Assurance in a Large Multi-Institutional Biorepository; The Experience of the PROCURE Quebec Prostate Cancer Biobank
}

\author{
Fadi Brimo, ${ }^{1}$ Armen Aprikian,, ${ }^{1,5}$ Mathieu Latour, ${ }^{2}$ Bernard Têtu, ${ }^{3}$ Alexandre Doueik, \\ Eleonora Scarlata, ${ }^{1}$ Lucie Hamel, ${ }^{1}$ Ginette McKercher, ${ }^{5}$ Fred Saad, ${ }^{2}$ Louis Lacombe, ${ }^{3}$ \\ Michel Carmel, ${ }^{4}$ and Simone Chevalier ${ }^{1,5}$
}

Well-characterized, high-quality fresh-frozen prostate tissue is required for prostate cancer research. As part of the PROCURE Prostate Cancer Biobank launched in 2007, four University Hospitals in Quebec joined to bank fresh frozen prostate tissues from radical prostatectomies (RP). As the biobank progressed towards allocation, the nature and quality of the tissues were determined. RP tissues were collected by standardized alternate mirror-image or biopsy-based targeted methods, and frozen for banking. Clinical/pathological parameters were captured. For quality control, two presumed benign and two presumed cancerous frozen, biobanked tissue blocks per case (10/site) were randomly selected during the five years of collection. In a consensus meeting, 4 pathologists blindly evaluated slides $(n=160)$ and graded quality, Gleason score (GS), and size of cancer foci. The quality of tissue RNA (37/40 cases) was assessed using the RNA Integrity Number. The biobank included 1819 patients of mean age: 62.1 years; serum PSA: $8 \mathrm{ng} / \mathrm{ml}$; prostate weight: $47.8 \mathrm{~g}$; GS: 7; and pathological stage: $\mathrm{T} 2$ in $64.5 \%$, T3A in $25.5 \%$ and T3B in $10 \%$ of cases. Of the 157 evaluable slides, 79 and 78 had benign and cancer tissue, respectively. GS for the 37 cancer-positive cases were: 6 in 9, 7 in 18 and $>7$ in 10 and, in most instances, in concordance with final GS. In $40 \%$ of slides containing cancer, foci occupied $\geq 50 \%$ of block surface and $42 \%$ had a diameter $\geq 1 \mathrm{~cm}$. Tissue was well preserved and consistently yielded RNA of very good quality with RNA Integrity Number $($ RIN) $>7$ for $97 \%$ of cases (mean $=8.7 \pm 0.7)$ during the five-year collection period. This study confirms the high quality of randomly selected benign and cancerous fresh-frozen prostate tissues of the PROCURE Quebec Prostate Cancer Biobank. These results strengthen the uniqueness of this large prospective resource for prostate cancer research.

\section{Introduction}

Prostate cancer remains the most common malignancy in men in the Western world. ${ }^{1}$ With the advent of PSA-screening and extended biopsy scheme techniques, a significant proportion of the detected cases may be indolent, low-stage cancer where patients may die with it rather than from it. However, certain subtypes of prostate cancer are remarkably aggressive with rapid progression to metastases and resistance to therapy. Also, a non-negligible proportion of clinically localized cases progresses to metastatic disease. ${ }^{2}$ Due to its high incidence, prostate cancer currently remains the second leading cause of cancer deaths following lung cancer. ${ }^{1}$ These points demonstrate the critical need to identify biomarkers of biologically aggressive cancers that correlate with a worse prognosis and that dictate the need for more aggressive treatment. In that regard, although major work has been undertaken in the last few years to characterize the molecular pathways involved in prostate cancer, the genetic and molecular changes associated with progression of the disease are still largely unknown. As our knowledge about the disease is gained primarily from molecular and genomic studies, the establishment of large biobanks composed of high-quality and well characterized prostate tissues has come to play a crucial role in the research field. This being said, in order to reach meaningful and comparable results from different studies using a specific cohort, the medical centers involved in the biobanking process must possess robust and validated biobanking protocols and regularly perform quality control tests to ensure high-quality samples. 
As part of the PROCURE Prostate Cancer Biobank project launched in the province of Quebec in 2007, the four University Hospitals Centres in the province (Montreal, McGill, Quebec, and Sherbrooke) have joined in an effort to build a prospective biobank of fresh-frozen benign and malignant prostate tissues from radical prostatectomies as well as clarified urine and blood samples from which serum, plasma, the buffy coat layer, RNA and DNA were also derived. As a quality control measure as the biobank is progressing towards allocating the samples, we assessed the nature and quality of the tissues and the quality of the RNA in a randomly selected set of cases collected over a five-year period obtained from the four participating centers.

\section{Materials and Methods}

Ninety-seven percent of patients who were asked to be part of the biobank prior to undergoing radical prostatectomy at the four centers accepted. Tissue, blood and urine samples were collected only from consented patients preoperatively. The blood and urine samples were processed and stored according to standard operating procedures used by each biobank site. The methods by which the fresh prostate tissues were harvested for biobanking and by which the prostate was sampled for histological assessment differed across the four centers according to the pathologist's preferred practice and to the logistic and technical characteristics specifically in place at each site. After inking the prostate, one site used the previously described "alternative slices mirror image protocol" in which the prostate is sliced from apex to base perpendicular to the prostatic urethra and each slice subsequently cut into four quadrants. ${ }^{3}$ Each quadrant is thinly sliced and alternatively submitted for histological assessment (paraffin embedded) and for biobanking in a mirror image fashion while ensuring that the prostate apex and base are submitted for histology. ${ }^{3,4}$ The term 'mirror image' refers to embedding the frozen block so that its free, first-cut surface corresponds to the cut surface of the immediately adjacent paraffin-embedded block. The other three centers used a "biopsy- and palpation-based targeted method of sampling." A whole cross-section of the prostate tissue was taken for biobanking and was frozen as four quadrants. The pathology report of the biopsies along with the gross appearance and palpation oriented the selection of the section in order to optimize the amount of tumor tissue present within the selected sample. The latter was then sectioned into multiple pieces and snap-frozen. The remaining prostate tissue was further sectioned at $5 \mathrm{~mm}$ intervals from the apical to the vesical margins and submitted in its entirety for histology. Grossly suspicious areas were identified as firm irregular nodules or areas with color or texture heterogeneity. Within 60 minutes after collection from the operating room, the samples were measured (length, width, thickness), placed in $30 \mathrm{~mm}$ disposable base molds, coated in OCT compound, snap-frozen in precooled isopentane in liquid nitrogen, and stored in a $-80^{\circ} \mathrm{C}$ freezer. Using the "mirror image" protocol the frozen blocks were numerically labelled and their corresponding paraffin-embedded blocks identified. Frozen samples were identified using a uniform labelling system across all sites with laminated tape using the $P$ touch PRO XL system that encodes for specimen type $(P$ : prostate), clinical site (S: 01-04), patient biobank number $(P$ : 0001-9999), and the frozen block number (Frz: 1-4); (e.g.,:
PS03P0001Frz1). Due to the previously reported high diagnostic correlation between the biobanked and paraffin-embedded blocks, the diagnosis in a given frozen block was presumed to be identical to its mirror image block. ${ }^{3}$ In the cases in which the 'biopsy- and palpation-based targeted method' of sampling was used, the area from which the frozen block was obtained was identified, and the nature of tissue in a given block presumed to be similar to the paraffinembedded tissue adjacent to it. Clinical and final pathological parameters were captured in the central biobank database using the ATiM (Advanced Tissue Management) software, which was developed as part of the Canadian Tumor Repository Network (CTRNet).

The quality control study included 40 cases, 10 from each center, randomly selected but covering the five-year collection period. Four frozen OCT blocks from each case were used for histological evaluation, two of which were presumed to contain benign prostate tissue and two presumed to contain cancerous tissue. These blocks were cut and the sections stained with routine hematoxylin-eosin (H\&E) staining. In a consensus meeting that included the four participating pathologists $(\mathrm{FB}, \mathrm{ML}, \mathrm{BT}, \mathrm{AD})$ from the four centers, the sections were evaluated blindly and the following parameters recorded: quality of tissue; nature of tissue (benign versus cancer); Gleason score using the modified Gleason grading system of $2005^{5}$; and percentage of the section occupied by cancer and the length of the largest focus of cancer. A consensus agreement was reached when three of the participating pathologists had concordant diagnoses. Following this meeting, the Gleason score of individual sections were compared to the final Gleason score in the pathology report.

For RNA extraction, left over tissue from cryostat sectioning of the four blocks from each case were pooled and transferred into one Eppendorf tube on ice containing $350 \mathrm{uL}$ of cold lysis buffer (RLT buffer, Qiagen), vortexed, and aspirated 5 times through a syringe with a $20 \mathrm{G}$ needle. RNA was then extracted with the RNeasy Micro Kit from Qiagen (Hilden, Germany). RNA concentration was measured using a NanoDrop 8000 spectrophotometer and RNA Integrity Number (RIN) determined using the Agilent 2100 Bioanalyzer with RNA 6000 Nano-Lab chip kit (Agilent Technologies, Santa Clara, CA, USA). ${ }^{6}$ Very good quality RNA was defined as having a $\mathrm{RIN} \geq 7$.

\section{Results}

At the time of quality assessment, the biobank included 1819 patients with complete clinical and pathological data available in the database for 1570 patients. The clinical and pathological characteristics of the cohort are presented in Table 1. Of note, none of the parameters were statistically different among the different sites (data not shown), reflecting similar populations, treatment modalities and histological diagnostic assessment.

A representative example of one of the blocks cut at the Cryostat is shown in Figure 1A.Mean size of the blocks was $1.41 \pm 0.36 \mathrm{~cm}$ (length) by $0.93 \pm 0.29 \mathrm{~cm}$ (width), with a thickness of $\sim 0.4 \mathrm{~cm}$. For every block, a slide was prepared, resulting in 160 slides corresponding to 40 cases. Three slides had significant artefacts that precluded assignment of a definitive benign or cancerous diagnosis. In three out of the 40 cases, none of the four selected slides showed cancer 
Table 1. Clinical and Pathological Characteristics of THE Biobank COHORT $(N=1570)$

Mean age (y)

Mean pre-operative PSA $(\mathrm{ng} / \mathrm{ml})$

Mean Prostate weight (g)

Final Gleason Score

6

7

8

9

10

Pathological Stage

T2

T3A

T3B

62.1

8

47.8

$\%$

25

64

6

5

0.1

$\%$

64.5

25.5

10

in the frozen blocks, representing a cancer detection rate of $92.5 \%$. Nineteen slides showed freezing artefacts in which it was still possible to assign a definitive diagnosis. Of the 157 evaluable slides, 79 contained benign prostate tissue and no evidence of invasive carcinoma (six contained high-grade prostatic intraepithelial neoplasia or HGPIN) and 78 showed invasive adenocarcinoma, all of which were of a quality that enabled assignment of a Gleason score. Despite the fact that half of the slides showed cancer corresponding to the 1:1 expected presumed benign to cancer ratio, the diagnosis in individual slides did not consistently correlate with the presumed diagnosis; while $32 \%$ of the presumed benign blocks turned out to contain invasive adenocarcinoma, $32 \%$ of the presumed cancerous blocks did not contain invasive carcinoma. Although there was no difference in the overall cancer detection rate in the four blocks across sites $(\approx 50 \%)$, the ability to predict the cancerous nature of tissue in a block was best using the 'alternative slices mirror image protocol' when compared to the other methods of sampling ( $84 \%$ versus 61\%; Fig.1B). Tumor foci in the examined banked blocks occupied a mean of $40.6 \%$ of the sections, and had a mean diameter of $8.4 \mathrm{~mm}$; the calculated banked volume of tumor per block $\left(\pi \mathrm{r}^{2} \mathrm{~h}\right)$ was $0.220 \mathrm{~cm}^{3}$ or $\mathrm{g}$ of tissue (assuming a density $=1 \mathrm{~g} / \mathrm{cm}^{3}$ ). Forty-two percent of the cancerous foci were $\geq 1 \mathrm{~cm}$ in size and in $40 \%$ of the sections, the foci covered $\geq 50 \%$ of the surface. The Gleason score attributed to the cancerous foci was 6 in $44 \%$, 7 in $32 \%$ and $8-10$ in $24 \%$ of the 78 slides in which cancer was detected. Of the 24 cases with a final Gleason score of 7 ,

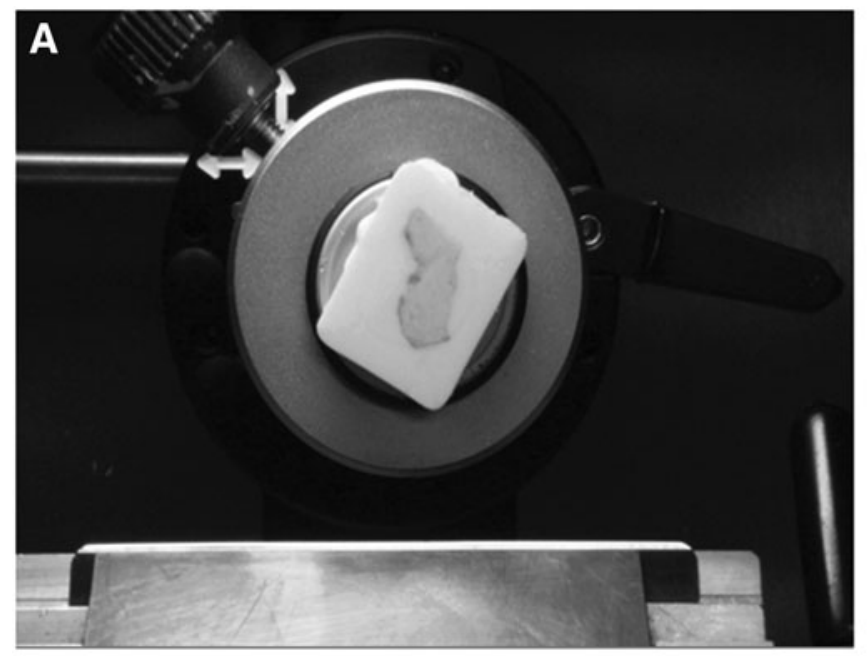

\section{B}
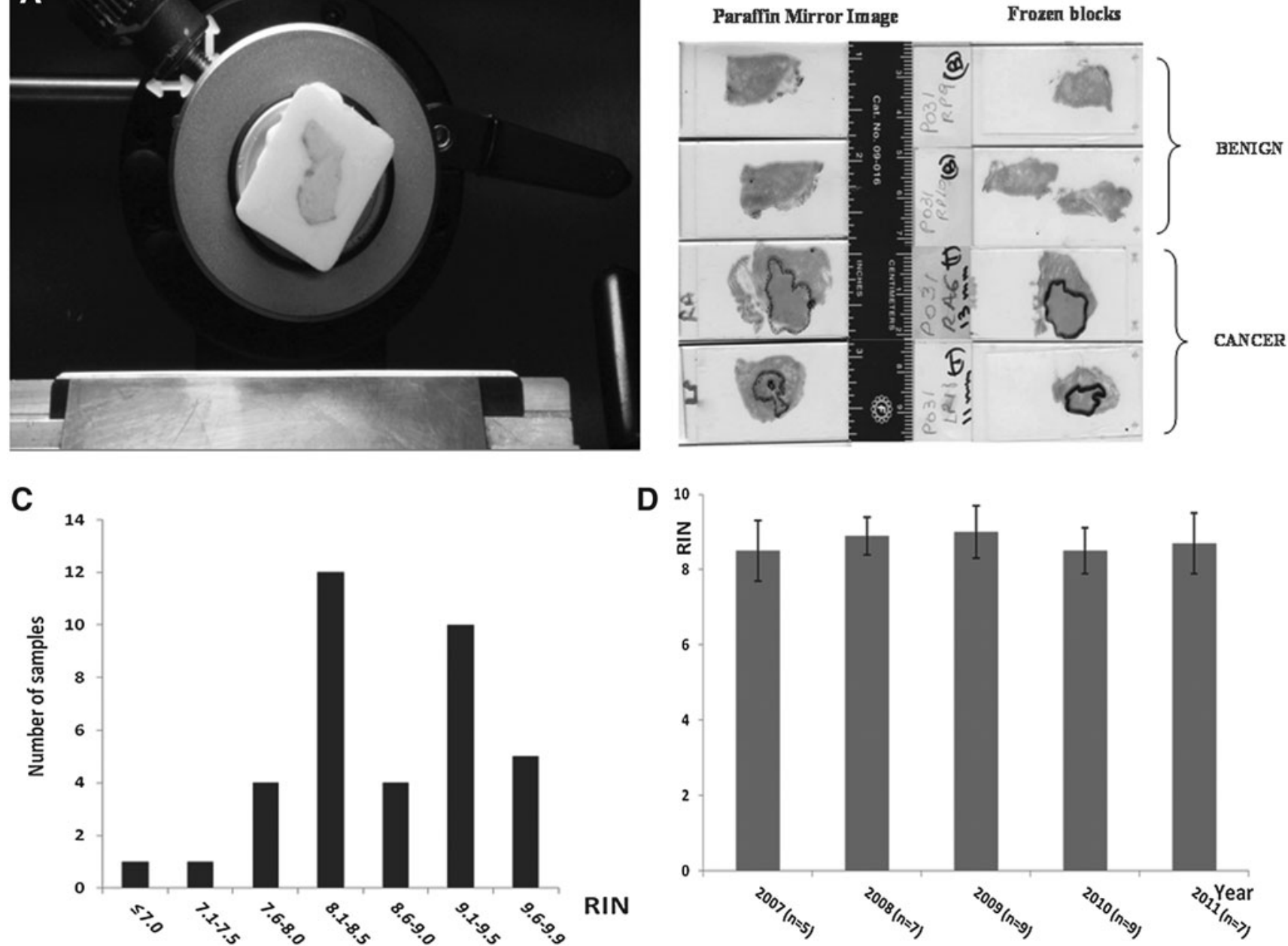

FIG. 1. (A) A frozen tissue block processed at the Cryostat; (B) Mapping of the cancerous regions of the frozen blocks; (C) RIN values of tissue RNA for 37 cases; (D) Mean RIN values of tissues banked from 2007 to 2011. 
Table 2. Correlation of the Gleason Score of Cancer in the Frozen Tissue with the Final Score After Radical Prostatectomy

\begin{tabular}{lccc}
\hline & \multicolumn{3}{c}{ Highest GS in frozen tissue } \\
\cline { 2 - 4 } RP GS & $6(\mathrm{n}=9)$ & $7(\mathrm{n}=18)$ & $8-10(\mathrm{n}=10)$ \\
\hline $6(n=5)$ & 5 & 0 & 0 \\
$7(n=24)$ & 4 & 18 & 2 \\
$8-10(n=8)$ & 0 & 0 & 8 \\
\hline
\end{tabular}

GS, Gleason score; RP, radical prostatectomy.

the frozen cancerous tissue was of lower grade $(G S=6)$ in four cases, and in two cases the frozen blocks contained cancer with a score of 8 , reflecting tumor heterogeneity. This represents sampling of an area purely composed of one Gleason grade/pattern (pattern 3 or 4 ) rather than representing a tumor component of a high grade/pattern (pattern 4 or 5) that was unsampled in the paraffinembedded tissue (Table 2). RIN values determined for tissue RNA extracted for the 37 cases analyzed are illustrated in Figure 1C. For 36 out of the 37 cases, RIN values were higher than 7. Moreover, $31 / 37$ cases had RIN values $\geq 8.0$, implying a high quality RNA. The mean RIN value obtained was $8.7 \pm 0.7$. The comparison of mean RIN values of RNA extracted from tissues collected yearly since 2007 showed no difference; as can be seen in Figure 1D, RIN values were constant over time, indicating good preservation of the banked frozen tissue collected.

\section{Discussion}

The PROCURE Prostate Cancer Biobank is a multi-institutional provincial effort that aims to accelerate research into the genetics and biology of prostate cancer by providing a large number of human fluids and prostate tissue specimens to different regional and international scientists. In order to ensure appropriate tissue quality before release for research, quality control measures were implemented and the results reviewed each year during the annual biobank meeting. The first goal of such measures was to document the composition of the biobanked tissue in order to ensure that the nature of the released tissue corresponds to the needs of a specific project. This goal can be achieved in either of two ways: by histological evaluation of a paraffinembedded tissue that is thought to correspond to the biobanked fresh frozen tissue, a process which limits wasting the frozen tissue for analysis; or by obtaining an H\&E section from the frozen tissue block, either at the time of harvesting or before the tissue allocation. Obtaining H\&E sections at the time of biobanking is logistically demanding, time consuming, and requires the presence of a pathologist which is not always possible. Therefore we evaluated slides obtained from paraffin-embedded blocks that are thought to match the diagnosis in the biobanked tissue. In the centers in which the palpation-based targeted method of sampling was used, the diagnosis in the paraffin-embedded tissue adjacent to the frozen tissue was recorded and correlated with that of the frozen tissue. When the alternative slices mirror image protocol was used, the mirror image paraffin blocks were examined. The ability to predict the presence of cancer in a given block was best using the alternative slices mirror image protocol when compared to the other method of sampling ( $84 \%$ versus $61 \%$ ), emphasizing the known fact that prostate cancer is frequently difficult to detect by gross inspection alone. ${ }^{7}$ Interestingly, $25 \%$ of the presumed benign frozen blocks turned out to be contaminated by cancer using the same method. This good but not excellent correlation rate highlights the need to characterize the nature of the biobanked tissue by evaluating H\&E sections obtained from all frozen blocks, a process that is currently being undertaken. Another advantage of such a process would be to document the presence of histological entities other than benign tissue or invasive adenocarcinoma, which could potentially affect study results. For example, HGPIN, which is known to harbor molecular changes that overlap with invasive carcinoma, was detected in 6/157 slides in the current study. Also, this would enable the identification of a potential minor high grade cancer component that was not sampled in the paraffin-embedded sections. However, none of the examined frozen tissue blocks in the current study $(n=157)$ as well as our previously related published study $(n=200)$ showed a high Gleason pattern that was not detected and reported in the routine pathological evaluation. Accordingly, the biobanking process did not preclude the assignment of an accurate Gleason score in a given case of our cohort. ${ }^{3}$

In regard to the biobanking method used, the current data suggests that there is no significant difference in the ability to bank cancerous tissue whether the biopsy- and palpationbased targeted method of sampling or the alternative slices mirror image protocol were used, as the success rate to bank cancer per case was excellent across all centers and ranged from $90 \%$ to $100 \%$. Of note is that the reported $92.5 \%$ yield of cancer biobanking applies only to the examined 4 blocks per case that were used for the quality control study, which makes it likely that the actual rate could differ and possibly be higher if all biobanked blocks of a given case were examined. This rate is comparable to the Weill Cornell Medical College institutional prostate biobank (cancer detection rate of $90 \%$ in 105 examined cases) in which a technique similar to the alternative slices mirror image protocol was used. However, in that study all the frozen blocks were examined in comparison to four blocks per case in this study. ${ }^{2}$ Alternative banking techniques using punch biopsies (rather than entire tissue blocks) obtained from grossly benign and suspicious areas of the fresh prostate have also been described. Using this method, the cancer detection rate in a study by Riddik et al. was $87 \%$ in prostatic transurethral resections and only $63 \%$ in radical prostatectomy specimens, which is not optimal when compared to our results or those of Esgueva et al. ${ }^{2,8}$

Following confirmation that the banked tissue meets high standards in terms of yield and quantity of cancer, and tissue quality at the histological level, examining the tissue RNA was essential in order to ensure that the material can be reliably used for gene expression or RNA-sequencing studies. In that effect the RNA integrity was shown to be an important factor that can impact downstream applications such as next generation sequencing and RT-PCR. ${ }^{9-11}$ Also, the presence of matching paraffin material in the biobank empowers such RNA-based studies by enabling immunohistochemical evaluation of genes of interest at the protein level. Different methods of assessing RNA quality exist, the 
most validated and currently widely used being the RIN, ${ }^{6}$ which is a software algorithm that allows the classification of total RNA, based on a numbering system from 1 (most degraded) to 10 (intact). ${ }^{11-15}$ Using the Agilent 2100 Bioanalyzer and lab-on-chip microfluid technology, the software generates an electropherogram. The RIN algorithm then generates its integrity number by taking into account the entire trace. Using this system, RNA of very good quality should have a RIN $\geq 7$. In the current study, we were able to demonstrate that 36 cases $(97 \%)$ had a RIN $>7$, with a mean of $8.7 \pm 0.7$. Moreover, we have also shown that tissues banked for more than five years had RIN values as good as recently banked tissues, indicating that prostate tissue block integrity is well preserved with time. In a similar study by Dev, et al., which included 186 prostate samples taken from 142 patients who underwent $\mathrm{RP}$, the authors demonstrated mean RINs of $4.91( \pm 1.67, n=47)$ for stromal cells, 6.76 $( \pm 1.45, n=66)$ for benign prostate tissue, and $7.7( \pm 1.46$, $n=73$ ) for prostate adenocarcinoma. ${ }^{12}$ In that study, $27 \%$ of epithelial samples were insufficient for high fidelity RNA studies and $73 \%$ of samples had RIN $>7 .{ }^{12}$ In comparison, Bertilsson et al. analyzed the RNA quality in 354 cores taken from 46 RPs and reported low quality RNA $(\mathrm{RIN}<4)$ in $19 \%$ of the cores with only $14 \%$ of the cores having RIN $\geq 7 .{ }^{16}$ On the other hand, Ricciradelli et al. were able to obtain RIN values of $8-10$ using only five prostate specimens. ${ }^{13} \mathrm{Al}-$ though prostate cancer RIN scores are lower in most previously reported studies than in the current one, it is interesting to note that all of the reported scores remain relatively high (most RINs $>6$ ) pointing to the possibility that prostate tissue may be more resistant to RNA degradation than other tissue types. As an example, RNA obtained from placental tissue immediately after delivery yielded RNA values ranging from 4.5 to 7.2 in one study. ${ }^{17}$ Also, a study performed using a Memorial Sloan-Kettering Cancer Center-based pancreatic cancer biobank that includes 64 cases reported that only $42 \%$ of samples had a RIN $\geq 7 .{ }^{18}$ While it is conceivable that cellular content affects degradability of the tissue leading to an impact on RNA integrity (pancreatic or placental tissue being more sensitive to degradation than prostate tissue), there is also some evidence suggesting that the release of RNase by intraoperative manipulation plays a role in RNA degradation. Therefore, biobanked specimens obtained by open surgery may be more prone to have lower quality RNA than those in which laparoscopic or robotic surgery is used. ${ }^{12,19}$ However, the human factor contribution to the variability in RNA quality cannot be excluded and has not been specifically addressed in various studies.

Taken together, this study confirms the high quality of randomly selected benign and cancerous fresh-frozen prostate tissues of the PROCURE Quebec Prostate Cancer Biobank both at the histological and RNA integrity levels. These results strengthen the uniqueness of this large prospective cohort and make it a valuable resource for prostate cancer research.

\section{Acknowledgments}

The authors acknowledge the Réseau de Recherche sur le Cancer supported by Le Fonds de la Recherche du Québec en Santé for RNA analyses. Biobanking was rendered possible by patient participation and through donations to PROCURE, coming from individuals as well as companies and to a one-time donation from Prostate Cancer Canada.

\section{Author Disclosure Statement}

The authors declare no conflict of interest.

\section{References}

1. Jemal A, Siegel R, Xu J, Ward E. Cancer statistics, 2010. CA Cancer J Clin 2010;60:277-300.

2. Esgueva R, Park K, Kim R, et al. Next-generation prostate cancer biobanking: toward a processing protocol amenable for the International Cancer Genome Consortium. Diagn Mol Pathol 2012; 21:61-68.

3. Brimo F, Sircar K, Chevalier S, et al. Banking of fresh-frozen prostate tissue using the alternate mirror image protocol: methods, validation, and impact on the pathological prognostic parameters in radical prostatectomy. Cell Tissue Bank 2012;13: 631-638.

4. Sircar K, Gaboury L, Ouadi L, et al. Isolation of human prostatic epithelial plasma membranes for proteomics using mirror image tissue banking of radical prostatectomy specimens. Clin Cancer Res 2006;12:4178-4184.

5. Epstein JI, Allsbrook WC, Jr., Amin MB, Egevad LL. The 2005 International Society of Urological Pathology (ISUP) Consensus Conference on Gleason Grading of Prostatic Carcinoma. Am J Surg Pathol 2005;29:1228-1242.

6. Lawson MH, Rassl DM, Cummings NM, et al. Tissue banking of diagnostic lung cancer biopsies for extraction of high quality RNA. J Thorac Oncol 2010;5:956-963.

7. Epstein JI. Radical prostatectomy: processing, staging, and prognosis. Parts I and II. Int J Surg Pathol;18:118S-123S.

8. Riddick AC, Barker C, Sheriffs I, et al. Banking of fresh-frozen prostate tissue: methods, validation and use. BJU Int 2003;91: 315-323; discussion 23-2-4.

9. Fleige $S$, Pfaffl MW. RNA integrity and the effect on the real-time qRT-PCR performance. Mol Aspects Med 2006;27:126-139.

10. Fleige S, Walf V, Huch S, Prgomet C, Sehm J, Pfaffl MW. Comparison of relative mRNA quantification models and the impact of RNA integrity in quantitative real-time RT-PCR. Biotechnol Lett 2006;28:1601-1613.

11. Schroeder A, Mueller O, Stocker S, et al. The RIN: an RNA integrity number for assigning integrity values to RNA measurements. BMC Mol Biol 2006;7:3.

12. Dev H, Rickman D, Sooriakumaran P, et al. Biobanking after robotic-assisted radical prostatectomy: a quality assessment of providing prostate tissue for RNA studies. J Transl Med 2011;9: 121-129.

13. Ricciardelli C, Bianco-Miotto T, Jindal S, et al. Comparative biomarker expression and RNA integrity in biospecimens derived from radical retropubic and robot-assisted laparoscopic prostatectomies. Cancer Epidemiol Biomarkers Prev 2010;19: $1755-1765$.

14. Strand C, Enell J, Hedenfalk I, Ferno M. RNA quality in frozen breast cancer samples and the influence on gene expression analysis-a comparison of three evaluation methods using microcapillary electrophoresis traces. BMC Mol Biol 2007;8: $38-46$.

15. Copois V, Bibeau F, Bascoul-Mollevi C, et al. Impact of RNA degradation on gene expression profiles: assessment of different methods to reliably determine RNA quality. J Biotechnol 2007; 127:549-559.

16. Bertilsson $H$, Angelsen A, Viset $T$, Skogseth $H$, Tessem MB, Halgunset J. A new method to provide a fresh frozen prostate slice suitable for gene expression study and MR spectroscopy. Prostate 2011;71:461-469. 
17. Fajardy I, Moitrot E, Vambergue A, Vandersippe-Millot M, Deruelle P, Rousseaux J. Time course analysis of RNA stability in human placenta. BMC Mol Biol 2009;10:21.

18. Rudloff U, Bhanot U, Gerald W, et al. Biobanking of human pancreas cancer tissue: impact of ex-vivo procurement times on RNA quality. Ann Surg Oncol 2010; 17:2229-2236.

19. Bertilsson H, Angelsen A, Viset T, Anderssen E, Halgunset J. RNA quality in fresh frozen prostate tissue from patients operated with radical prostatectomy. Scand J Clin Lab Invest 2010;70: 45-53.
Address reprint requests to: Fadi Brimo, M.D. Department of Pathology McGill University Health Centre Montreal General Hospital 1650 Cedar Avenue Montreal, Quebec, H3G 1A4 Canada

E-mail: fadi.brimo@muhc.mcgill.ca 